CDR3 and correct for sequencing errors. Quantification was validated in a dilution series of Jurkat $T$ cells in a background of human CD4 T cells. Validation against the 'gold standard' of cloning and sequencing was performed in murine models of flu infection and ovalbumin immunisation using tetramer responding $T$ cells as read-out.

Results The technique successfully yielded the sequences of the $\mathrm{V}$ and $\mathrm{J}$ segments and the CDR3 region in all repertoires. The human TCR analysis recovered 13/13 J and 47/49 functional $\mathrm{V}$ genes in a HD blood sample. In a murine blood sample, $13 / 13 \mathrm{~J}$ and $22 / 22 \mathrm{~V}$ genes were identified. The BCR analysis showed $6 / 6 \mathrm{~J}$ and $44 / 44 \mathrm{~V}$ genes in blood from a HD. The dilution experiment showed a linear relation between input (Jurkat/CD4) and output (Jurkat/CD4) $\left(\mathrm{R}^{2}=99, \mathrm{p}<0.0001\right)$. In the flu and ovalbumin models, all clonal expansions found by cloning and sequencing (153 reads) in individual mice were also recovered in the HTS protocol (6825 reads) in approximately the same distributions.

Conclusions Our new HTS protocol overcomes current limitations in TCR/BCR repertoire analysis. It provides rapid unbiased quantitative information about complete repertoires at once in humans and mice. Clonally expanded cells could easily be identified in a background of resting cells for all repertoires analysed, both in blood and synovial tissue.

\title{
A81 HIGH THROUGHPUT SEQUENCING (HTS) PROVIDES FULL REPERTOIRE ANALYSIS OF THE B AND T CELL RECEPTORS IN HUMANS AND MICE, BOTH IN BLOOD AND SYNOVIAL TISSUE
}

P L Klarenbeek, ${ }^{1}$ P P Tak, ${ }^{1}$ M E Doorenspleet, ${ }^{1}$ B D C van Schaik, ${ }^{2}$ F M Wensveen, ${ }^{3} \mathrm{~L}$ Gottschal, ${ }^{1}$ M E Jakobs, ${ }^{4}$ I A M Derks, ${ }^{3}$ E Eldering, ${ }^{3}$ A H C van Kampen, ${ }^{2}$ F Baas, ${ }^{4} \mathrm{~N}$ de Vries ${ }^{1}{ }^{1}$ Department of Clinical Immunology and Rheumatology, AMC/University of Amsterdam, The Netherlands; '2Department of Clinical Epidemiology, Biostatistics and Bioinformatics, AMC/University of Amsterdam, The Netherlands; ' ${ }^{\text {Laboratory for }}$ Genome Analysis, AMC/University of Amsterdam, The Netherlands; ${ }^{4}$ Department of Experimental Immunology, AMC/University of Amsterdam, The Netherlands

10.1136/ard.2010.1296190

Background The adaptive immune system protects the body from invading pathogens. However, adaptive responses have also been implicated in autoimmune diseases such as rheumatoid arthritis. During these immune responses, activated $\mathrm{T}$ and $B$ cells can undergo clonal expansion. Identification and monitoring of expanded clones has proved difficult owing to the extremely high diversity of $\mathrm{T}$ and $\mathrm{B}$ cell receptor (TCR/ $\mathrm{BCR}$ ) repertoires. Current techniques are unable to rapidly and quantitatively analyse these vast repertoires with sufficient resolution; furthermore, they are vulnerable to artefacts. Here the authors show that high throughput sequencing (HTS) overcomes current limitations and can be successfully applied for TCR and BCR repertoire analysis in humans and mice, both in blood and synovial tissue.

Methods Primer sets were developed for the complete human TCR and BCR repertoires and murine TCR repertoire. The primers cover all functional $\mathrm{V}$ (ariable) and J(oining) genes ( $\beta$-chain (TCR), heavy-chain (BCR)), thereby spanning the CDR3, which was used to identify individual clones. A multiplex linear amplification was developed to provide unbiased amplification of all receptor $m$ RNA molecules. Peripheral blood mononuclear cells from two healthy donors (HD) and six Bl6 mice were analysed on a Genome Sequencer-FLX (Roche) providing $>100000$ receptor sequences per run. Bioinformatics tools were developed to identify gene segments, identify the 